\title{
Vom Tabu zum Thema
}

\section{Daniel Kauz}

Mit diesem Thema befasst sich auch das «Zu Guter Letzt» auf Seite 642.
Korrespondenz: lic. phil. Daniel Kauz Fokus AG für Wissen und Organisation Wattstrasse 3 CH-8050 Zürich

daniel.kauz@fokus-ag.ch www.fokus-ag.ch
Zu ihrem 100. Geburtstag hat die Krebsliga Schweiz den Historiker Daniel Kauz beauftragt, ihre Geschichte in einem Buch zusammenzufassen. Herausgekommen ist ein ansehnliches Werk über die schweizerische Krebsbekämpfung - auch im Umfeld der Krebsliga - mit Themen wie Aufklärungsarbeit, Fürsorgetätigkeit, Krebsstatistik, Grundlagenforschung und klinische Forschung. Die aktive Anbindung an Geschichtswissenschaft und Medizingeschichte verleiht der Jubiläumsschrift fachliche Seriosität. Der Band ist aufwendig hergestellt und prächtig illustriert. Als Bonus liegt eine DVD bei mit einem Aufklärungsfilm der Liga aus dem Jahr 1953: Prädikat sehenswert. Als "Appetithappen» folgen weiter unten einige Ausschnitte aus dem Buch, in denen es um das Sprechen und Schweigen über die Krankheit und Krebs als Tabu geht; ebenso im "Zu guter Letzt» auf Seite 642. (die Red.)

Der Chefarzt der Heilstätte Barmelweid, Max Buser, konstatierte 1956: «Der Begriff «Krebs` hat für uns alle etwas Unheimliches, Unbekanntes und Unheildrohendes. Wenn ein Mensch an einem Krebs leidet, versuchen oft wir Ärzte und die Angehörigen, ihm die Diagnose zu verheimlichen, weil wir alle unter der be- drückenden Tatsache stehen, dass ungefähr 82 Prozent der an Krebs Erkrankten ungeheilt bleiben.»

Das Nichtsprechen über Krebs bestand gleichsam aus einem Kreislauf von Verheimlichung und Misstrauen. Es handelte sich dabei um ein Tabu, das seitens des medizinischen und pflegerischen Personals freimütig eingestanden und diskutiert wurde. Bis in die zweite Hälfte des 20. Jahrhunderts schien es beinahe integraler Bestandteil der Krebsbehandlung zu sein. Die Tabuisierung der Erkrankung hatte zahlreiche kommunikative Ausprägungen, die von verschleiernden Umschreibungen und Beschönigungen über «gnädige Lügen», Gesprächsvermeidungen bis hin zu gefälschten Histologieberichten reichten. Lange Zeit war die Erkrankung in besonderem Masse mit dem Unheilbaren, Schicksalhaften, Unheimlichen assoziiert. Dieser Nimbus, die prekären Heilungsaussichten und eine weitgehend im Dunkeln liegende Ätiologie trugen wesentlich zur Tabuisierung bei. Während andere Krankheiten, wie die über Jahrhunderte gefürchteten Infektionskrankheiten oder die in den Industrienationen dominierenden HerzKreislauf-Erkrankungen, in ihren Ursachen bekannt,

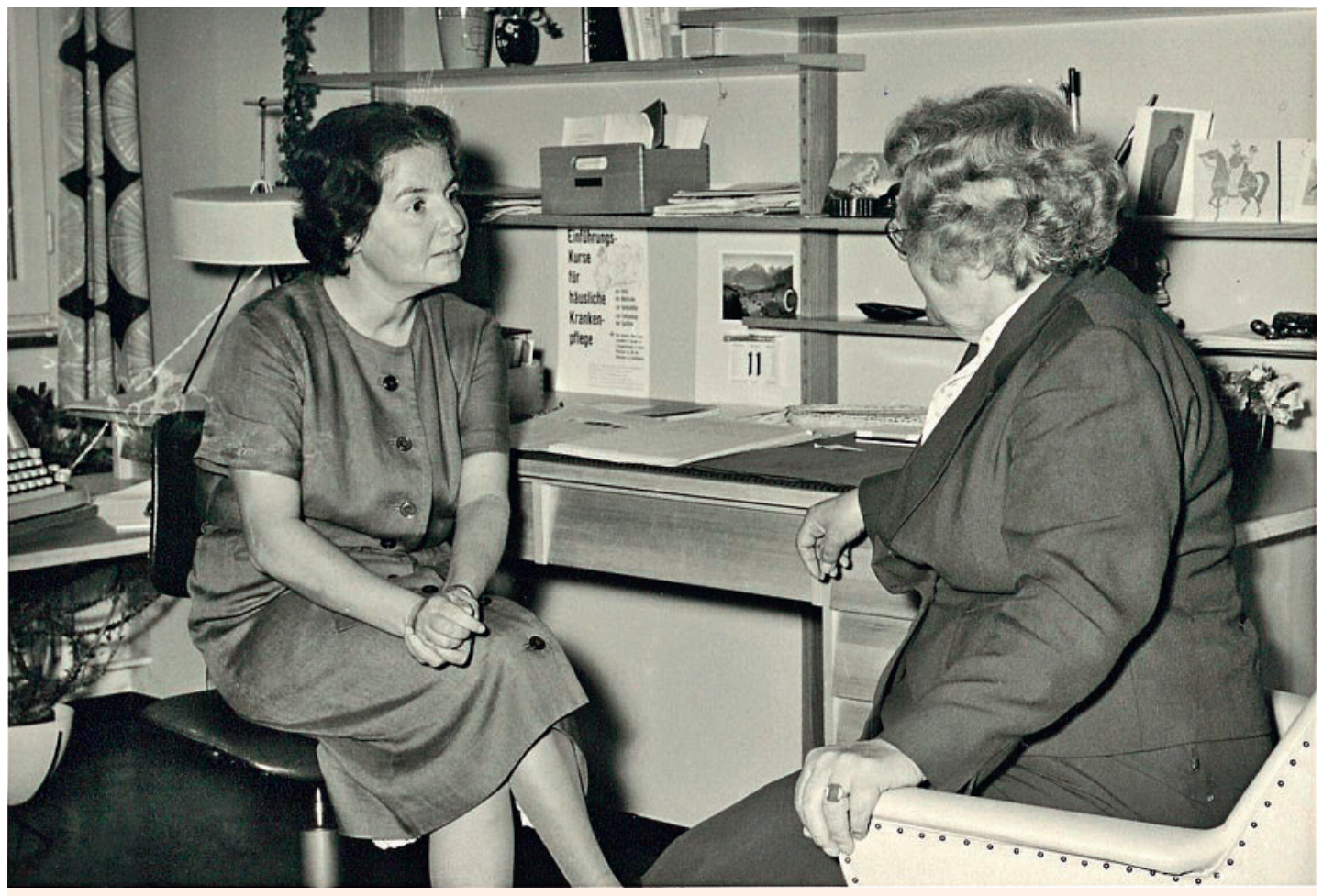

Beratungsgespräch mit der ersten Fürsorgerin der Krebsliga Zürich, Liselotte Hilb (links), vermutlich um 1963. Quelle: Archiv für Zeitgeschichte ETH Zürich (Nachlass Liselotte Hilb). 
immer besser behandelbar, teils gar «kontrollierbar» wurden, blieb vieles rund um die Karzinomerkrankungen unklar.

Die geringen Erfolgschancen einer Behandlung unterminierten auch das Bild des Arztes als «Heilender». Die Tabuisierung der Krebserkrankung war in der Schweiz, verglichen mit anderen europäischen Ländern oder den USA, wo zentrale Krebskliniken existierten, besonders ausgeprägt. Doch bestanden auch hier auffällige Unterschiede etwa zwischen der Deutschschweiz (mit einem sehr ausgeprägten Krebstabu) und der Romandie. Seit den 1970er Jahren setzte sowohl medizinintern wie auch seitens der Patienten eine Enttabuisierung des Sprechens über Krebs ein. Diese stellte einen wichtigen Schritt auf dem Weg zur Anerkennung des Patienten als Subjekt und seiner Bedürfnisse dar. Heutzutage ist «Patientennähe» zu einem zentralen Begriff im Selbstverständnis der Krebsliga geworden; über diesen definiert und legitimiert sie sich in einem bedeutenden Masse.

Die zur Zeit der Gründung der kantonalen Ligen und ihrer Beratungsstellen (ab den 1960er Jahren) alltägliche Tabuisierung von Krebs bestimmte den Umgang der Fürsorgerinnen mit den Betroffenen und deren Angehörigen noch massgeblich. Die Fürsorgerin der Berner Beratungs- und Hilfsstelle für Geschwulstkranke, Helen Zuppinger Matter, verwies auf die «sehr komplexen und typischen psychologischen Probleme» im Zusammenhang mit Krebs. Für den Spezialarzt erweise es sich, so Zuppinger Matter, «als äusserst schwierig zu beurteilen, welcher von den Angehörigen über den Zustand des Patienten orientiert werden kann». Aufgabe der Fürsorgerinnen - als Verbindungspersonen zwischen Ärzten, Betroffenen und Angehörigen - war es demnach auch, das Verheimlichen der Krankheit zu organisieren. Denn die Ungewissheit sah man zugleich als Garant der Hoffnung an: «Obwohl die meisten Patienten die Art ihrer Erkrankung ahnen, muss um jeden Preis vermieden werden, dass sie darüber Gewissheit erhalten. Solange der kleinste Zweifel möglich ist, bleibt auch die Hoffnung bestehen. Eine auf Krebspatienten spezialisierte

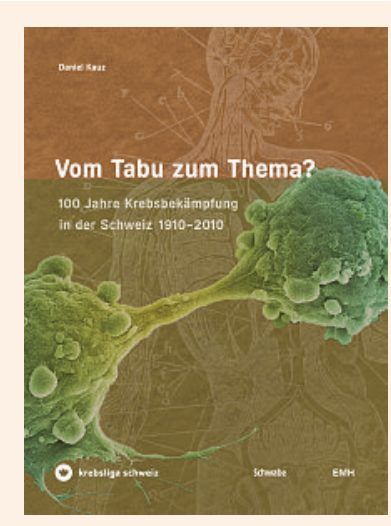

Krebsliga Schweiz (Hrsg.)

Daniel Kauz (Autor)

\section{Vom Tabu zum Thema?}

100 Jahre Krebsbekämpfung in der Schweiz 1910-2010. Basel: EMH Schweizerischer Ärzteverlag; 2010. 267 Seiten, 149 häufig farbige Abb. Mit DVD. 58 CHF ISBN 978-3-7965-2671-8.

(Auch auf Französisch unter dem Titel: Du tabou au débat? Cent ans de lutte contre le cancer en Suisse: 1910-2010. Hauterive: Ed. Attinger.)
Institution müsste daher unbedingt ihre Anonymität zu wahren suchen.» Auch die Fürsorgerin der Krebsliga Zürich hielt in ihren Erinnerungen fest, dass das Wort «Krebs» unter allen Umständen zu vermeiden war und stattdessen stets von «Geschwulst» gesprochen wurde.

\section{Man sah die Ungewissheit als Garant der Hoffnung an.}

Ein striktes Geheimhaltungsregime, wie es an der Berner Beratungs- und Hilfsstelle für Geschwulstkranke praktiziert wurde, durfte und konnte sich jedoch nicht allein auf kommunikative Strategien des Verheimlichens beschränken. Die konsequente Anonymisierung der Tätigkeit wurde sogar praktischinstitutionell umgesetzt: Die Halbtagsstelle der Krebsfürsorgerin war mit der Halbtagsstelle einer allgemeinen Fürsorgerin am Inselspital kombiniert. Dabei handelte es sich um ein Anstellungskonstrukt, das ausschliesslich geschaffen worden war, um den Betroffenen und ihren Angehörigen den Anschein zu geben, dass sie der regulären Spitalpflege zugewiesen worden seien. Die in Bern aufgebaute Beratungsstelle operierte somit gleichsam klandestin; selbst der Absender der Korrespondenz trug lediglich das nur schwer aufschlüsselbare Kürzel «B.H.G.» oder eine «neutrale Adresse».

Im kommunikativen Umgang mit Krebspatienten bestanden in der Schweiz dennoch unterschiedliche Kulturen, wie folgende Ausführungen der am Centre anticancéreux romand in Lausanne beschäftigten Fürsorgerinnen zeigen: «Ist es nicht so, dass es - vom psychologischen Standpunkt aus gesehen - zwei grosse Kategorien von Krebskranken gibt? Erstens alle die, welche die Tatsache, dass sie an Krebs leiden könnten, einfach unbewusst verdrängen. Sie klammern sich an jeden Strohhalm, der ihnen helfen kann, auch nur den kleinsten Hoffnungsschimmer aufrechtzuerhalten. Daneben gibt es aber auch alle die Patienten, die sich nicht täuschen lassen, was ihnen auch gesagt wird. Meistens sind diese Kranken innerlich auch stark genug, die Tatsache ihres Krebsleidens zu ertragen. Verschiedene Erlebnisse mit Patienten lassen darauf schliessen, dass die zweite Kategorie grösser ist, als allgemein angenommen wird. Die verschiedenen Kliniken für Krebskranke, die überall im Ausland existieren, zeigen ja übrigens auch, dass das durch die immer grösser werdende Spezialisierung entstandene Problem der Markierung der Krebskranken lösbar ist.» Ein Grund für die hier erkennbare offenere Haltung liegt sicherlich in der damals bereits seit Jahrzehnten bestehenden Tradition der Centres anticancéreux in der Romandie. 